\section{AB0606 NEUROPSYCHIATRIC LUPUS IN A SAMPLE OF EGYPTIAN PATIENTS WITH SYSTEMIC LUPUS ERYTHEMATOSUS: PREVALENCE AND CLINICAL CHARACTERISTICS}

M. Eissa, B.M. Medhat, A. Moghazy. Department of rheumatology and rehabilitation, Cairo University, Cairo, Egypt

Background: Neuropsychiatric (NP) lupus is common among patient with systemic lupus erythematosus (SLE). It occurs in about $30 \%-56 \%$ of all SLE patients. However, the diagnosis of neuropsychiatric SLE (NPSLE) remains difficult. Neuropsychiatric lupus (NPL) can present with a wide variety of clinical manifestations

Objectives: The aim is to determine prevalence of NPSLE among a sample of Egyptian SLE patients from a single centre and to describe its features and characteristics.

Methods: The study included 301 adult SLE patients from Cairo University Hospital. The patients are classified according to the Systemic Lupus International Collaborative Clinics (SLICC) criteria for SLE. Neuropsychiatric manifestations were recorded using the ACR NPSLE nomenclature and case definitions (1999) Global disease activity was quantified by the SLE Disease Activity Index 2000 (SLEDAI$2 \mathrm{~K}$ ) at first and at last visit of the patient. Systemic Lupus International Collaborative Clinics/ACR Damage Index (SLICC/ACR-DI) was used to measure damage. The period of data collection took 4 months. The collected data included demographic, clinical, serologic data and medications.

Results: 301 SLE patients $(87.4 \%)$ females and $(12.6 \%)$ males with mean age $30.7 \pm 9.2$ years and disease duration 72 months $(2-288)$ were included. 101 $(33.5 \%)$ were diagnosed as having NPSLE. The highest NP manifestation in frequency is headache $(55.4 \%)$ followed by psychosis $(33.7 \%)$ then seizures $(21.8 \%)$. NP manifestation is the onset of the disease in $42.6 \%$ of all NPSLE patients. Compared to non-NPSLE group, NPSLE group is significantly older a onset of disease and have longer disease duration $(\mathrm{p}<0.05)$. They are significantly more active at the onset of the disease than non-NPSLE and have significantly more disease damage $(p<0.05)$. Regarding clinical manifestations of lupus; NPSLE are significantly higher in frequency of discoid rash, cutaneous vasculitis, serositis, secondary anti-phospholipid syndrome (APS), associated avascular necrosis of the joints and osteoporosis $(\mathrm{p}<0.05)$. Anti-cardiolipin IgM anti bodies are significantly more frequent in NPSLE group $(p<0.05)$. Notably, frequency of psychosis, superior sagittal thrombosis and cerebrovascular disease were significantly higher in NPSL with positive APS than those with negative APS $(p<0.05)$ Conclusions: NPL is common in SLE, its prevalence is about $30 \%$ in Egyptian SLE patients. NPSLE patients may present diverse clinical manifestations most commonly headache, psychosis and seizures. NPL is different from non-NPL and presence of APS has an impact on clinical presentation of NP involvement in the patients.

Disclosure of Interest: None declared

DOI: 10.1136/annrheumdis-2018-eular.6883

\section{AB0607 CLINICAL PROFILE OF FILIPINO PATIENTS WITH SYSTEMIC LUPUS ERYTHEMATOSUS}

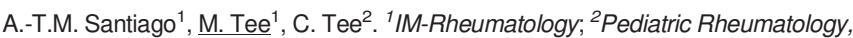
University of the Philippines Philippine General Hospital, Manila, Philippines

Objectives: To determine the clinical profile of Filipino SLE patients as determined using the 2012 Systemic Lupus Collaborating Clinics (SLICC) criteria. Methods: We checked which among the SLICC criteria were fulfilled by Filipino SLE patient when we examined them and their case records, as part of a nationwide genetic study on SLE conducted from October 2015 to March 2017.

Results: Two hundred twenty-five patients who were diagnosed to have SLE after 18 years of age were evaluated. The mean age of the population at the time of evaluation was 37.76 years.

Females comprised $98 \%$ of our population. Mean age of onset was 30.68 -yearold, while the mean age at diagnosis was 31.52-year-old. Acute cutaneous rash was found in $82 \%$; arthritis

$80 \%$; non-scarring alopecia $65 \%$; oral ulcers $52 \%$; renal disorder $41 \%$; chronic cutaneous rash in

$28 \%$; leukopenia $23 \%$; hemolytic anaemia $17 \%$; thrombocytopenia $16 \%$; serositis $13 \%$; and neurologic disorder $6 \%$. Anti-nuclear antibody was present in $88 \%$; low complement 33\%; antidsDNA in 29\%; direct Coombs' 4\%; antiphospholipid antibody $2 \%$; and anti-Smith antibody in

$2 \%$. Kidney biopsy was performed in only $6 \%(15 / 225)$ of patients, $33 \%$ of whom had Class IV histopathologic results.

Conclusions: Filipino SLE patients typically present with acute cutaneous rash, arthritis, nonscarring alopecia and oral sores. Renal disorder, hematologic and neurologic manifestations are found to be less common among them. Finally, it was noted that renal biopsy is not commonly performed among these patients.

Disclosure of Interest: None declared

DOI: 10.1136/annrheumdis-2018-eular.6108

\section{AB0608 CLINICAL PROFILE OF FILIPINO PAEDIATRIC PATIENTS WITH SYSTEMIC LUPUS ERYTHEMATOSUS}

A.-T.M. Santiago ${ }^{1}$, M. Tee ${ }^{1}$, C. Tee ${ }^{2} .{ }^{1} M$-Rheumatology; ${ }^{2}$ Pediatric Rheumatology, University of the Philippines Philippine General Hospital, Manila, Philippines

Background: The clinical profile of paediatric SLE patients may present differently from that of adults.

Objectives: To determine the clinical profile of Filipino paediatric SLE patients as determined using the 2012 Systemic Lupus Collaborating Clinics (SLICC) criteria. Methods: We checked which among the SLICC criteria were fulfilled by Filipino paediatric SLE patient when we examined them and their case records, as part of a nationwide genetic study on SLE conducted from October 2015 to March 2017.

Results: Ninety-eight who were diagnosed to have SLE before 18 years of age were evaluated.

The mean age of the population at the time of evaluation was 19.77 years. Females comprised

$94 \%$ of our population. Mean age of onset was 14.42 -year-old, while the mean age of diagnosis was at 14.58 -year-old. Acute cutaneous rash was found in $87 \%$; oral ulcers $65 \%$; renal disorder

$65 \%$; non-scarring alopecia $61 \%$; arthritis $58 \%$; chronic cutaneous rash in $37 \%$; leukopenia $36 \%$; hemolytic anaemia $34 \%$; serositis $24 \%$; thrombocytopenia $22 \%$ and neurologic disorder $8 \%$.

Anti-nuclear antibody was present in $88 \%$; low complement $33 \%$; anti-dsDNA in $28 \%$; direct

Coombs' 16\%; antiphospholipid antibody 3\%; and anti-Smith antibody in 1\%. Kidney biopsy was performed in only $14 \%(15 / 104)$ of patients, of whom $27 \%$ had class III histopathologic characteristic.

Conclusions: Filipino paediatric SLE patients typically present with cutaneous, mucocutaneous renal and musculoskeletal involvement. Hematologic and neurologic manifestations are found to be less common among them. Finally, it was noted that renal biopsy is not commonly performed among these patients.

Disclosure of Interest: None declared

DOI: 10.1136/annrheumdis-2018-eular.6661

\section{AB0609 CORRELATION BETWEEN PLASMA LIPOPROTEIN PHOSPHOLIPASE A2 (LP-PLA2) AND ANTI PHOSPHO LIPID ANTIBODY(APL-AB) IN PATIENTS WITH CONNECTIVE TISSUE DISEASES}

M. Yang, Z. Lin, X. Zhang, Q. Lv, Y. Zhang, Y. Jiang, Z. Liao, J. Gu. Third Affiliated Hospital of Sun Yat-Sen University, Guangzhou, China

Background: Plasma Lp-PLA2 has both anti-inflammatory and pro-inflammatory activity in vascular-related pathologies and is an independent risk predictor for coronary heart disease and stroke because of its important role in atherosclerosis and thrombosis. However, vasculitis is a common pathological feature of connective tissue disease.

Objectives: Therefore, the aim of this study was to investigate the association of plasma Lp-PLA2 level with antinuclear antibody (ANA) titer, karyotype, anti-cardiolipid antibody (ACA) and anti-B2GP1 antibody and lupus anticoagulant (LA including PT-IgG and PT-IgM)

Methods: Outpatient and inpatient with connective tissue diseases from Department of Rheumatology of the Third Affiliated Hospital of Sun Yat-sen University from 2015 to 2016 were randomly selected. Venous blood was collected, plasma Lp-PLA2 level was measured by Elisa method. ANA titer and karyotype were detected by indirect immunofluorescence assay. ACA, anti-B2GP1 antibody, PT $\mathrm{IgG}$ and PT-IgM were detected by Elisa method. SPSS23.0 statistical software was used for statistical analysis.

Results: A total of 176 patients with connective tissue disease including 38 males $(21.59 \%)$ and 138 females $(78.41 \%)$ were enrolled in this study, and the average age was $42.22 \pm 17.31$ years. The mean plasma level of Lp-PLA2 was (363.96 $\pm 203.19 \mathrm{ng} / \mathrm{ml}$ ). There was no significant difference in Lp-PLA2 level between different genders $(p=0.072)$, and LP-PLA2 level have no correlation with age $(p=0.098)$. The ANA titers were classified as negative $(35.80 \%)$, weakly positive 1: $100(11.93 \%)$, positive 1: $100(18.75 \%)$, positive 1: $320(12.50 \%)$, positive 1 : 1000 and positive $1: 3200(9.66 \%)$. There was no significant difference in plasma Lp-PLA2 level between different titers of ANA $(p=0.088)$. ANA karyotypes 